studies is not fully functional, although this is not proven. An alternative explanation is that at synapses and in cells, full-length sytI (an integral membrane protein) is needed not only for exocytic fusion, but also to initiate vesicle docking to the plasma membrane through binding to SNAP25. In this case, inhibition by the cytosolic domain could be explained by an unproductive interaction with the t-SNARE that prevents binding of full-length sytI (refs 9, 10). The reconstituted bilayers, however, could get into close proximity with each other more readily by bypassing the initial docking step. In this case sytI would function downstream of the process inhibited by the soluble sytI, because unlike in synaptic terminals, SNAP25 is already in a complex with syntaxin in the in vitro assay ${ }^{2}$. Therefore, accurate binding of fulllength sytI to the t-SNARE complex could be disturbed, whereas binding of the calciumactivated sytI domain would be sufficient to promote SNARE zippering and fusion (Fig. 2).

In conclusion, the results from Tucker et al. ${ }^{1}$ are an important milestone in the reconstitution of presynaptic events that result in neurotransmitter release. On the basis of these results, the need for proteins and lipids other than sytI in the calcium regulation of synaptic vesicle fusion ${ }^{5,6}$ can finally be excluded. Exocytic fusion, however, is a complex process consisting of a series of steps that can all be regulated. Previous evidence ${ }^{5}$ suggested that calcium-independent stimulation by sytI promoted assembly of the fusion-committed SNARE bundle. Whether this is the step at which calcium-dependent stimulation by sytI also occurs is unclear. Further studies with the bilayer reconstitution system may enhance our knowledge of the various steps in the fusion process and their regulatory mechanisms. This might ultimately result in the identification of other factors that regulate the system, in particular those allowing membrane fusion to operate in the sub-millisecond timescale as it does in synaptic physiology. $\square$

1. Tucker, W. C., Weber T \& Chapman, E. R Science 304, 435-438 (2004).

2. Weber, T. et al. Cell 92, 759-72 (1998).

3. Geppert, M. et al. Cell 79, 717-727 (1994).

4. Ubach, J. et al. EMBO J. 17, 3921-3930 (1998)

5. Mahal, L. K., Sequeira, S. M., Gureasko, J. M. \& Sollner, T. H. J. Cell Biol. 158, 273-282 (2002).

6. Avery, J., Jahn, R. \& Edwardson, J. M. Annu. Rev. Physiol. 61, 777-807 (1999).

7. Chapman, E. R. Hanson, P. I., An, S. \& Jahn, R. J. Biol. Chem. 270, 23667-23671 (1995).

8. Gerona, R. R., Larsen, E. C., Kowalchyk, J. A. \& Martin, T. F. J. Biol. Chem. 275, 6328-6336 (2000).

9. Zhang, X. et al. Neuron 34, 599-611 (2002).

10. Chieregatti, E., Witkin, J. W. \& Baldini, G. Traffic 3, 496-511 (2002).

\title{
Tip-ping off the COPs
}

Maintaining directionality is a critical aspect of vesicle transport: once vesicles have budded from an organelle, they are unable to fuse back to the donor membrane. But what determines this directionality? Reporting in the April 9 issue of Science, Faustin Kamena and Anne Spang now show evidence for an active mechanism that prevents back-fusion of vesicles to their donor organelle.

COPII proteins coat vesicles involved in endoplasmic reticulum-toGolgi (anterograde) transport, whereas COPI proteins coat vesicles involved in Golgi-to-endoplasmic reticulum (retrograde) transport. Kamena and Spang reasoned that proteins involved in COPI vesicle fusion might also recognize COPII vesicles and actively prevent backfusion. To test this, they examined a range of temperature-sensitive yeast mutants with defects in the early secretory pathway using an in vitro 'round-trip' assay. This assay recapitulates one complete cycle of transport from the endoplasmic reticulum through the Golgi and back to an acceptor endoplasmic reticulum. The addition of fresh acceptor endoplasmic reticulum to the assay means that a radiolabelled marker protein enclosed in COPII vesicles can be used to identify whether these vesicles have back-fused.

In mutants of tip20-8, back-fusion of COPII vesicles with the ER was observed at the non-permissive temperature. Although the efficiency of COPII vesicle fusion with the Golgi was reduced in tip208 mutants at the non-permissive temperature in whole cells, Kamena and Spang showed that the vesicles would fuse with purified Golgi in vitro. As anterograde transport is functional in tip20-8 mutants, it may be that the vesicles are not reaching the Golgi. Thus, Tip20 emerged as a good candidate for a factor that permits back-fusion of COPII vesicles.

To test this theory, Kamena and Spang shortened the round-trip assay so that COPII vesicle budding could occur, but vesicles did not have sufficient time for fusion with the Golgi. In this case, no marker protein was detected in the acceptor endoplasmic reticulum of wild-type cells, whereas $3-5 \%$ was detected in tip20-8 mutants. Mutants of Sec20p — which interacts with Tip20 for COPI vesicle

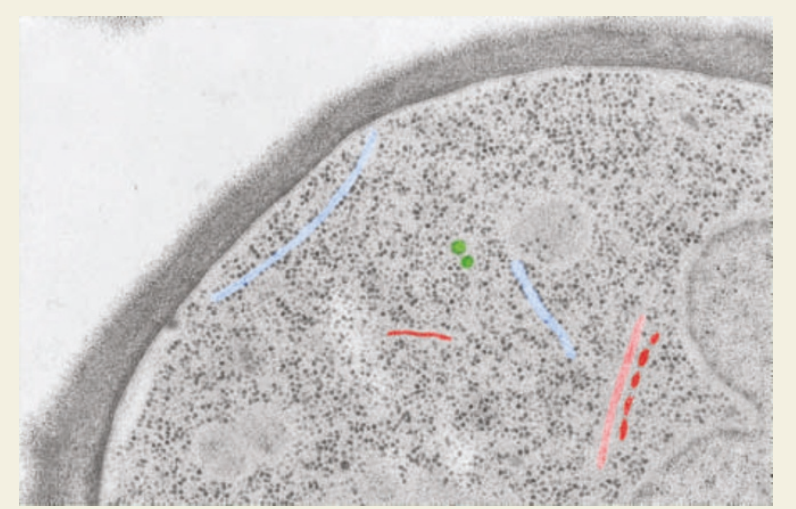

An electron micrograph showing part of a yeast cell. The endoplasmic reticulum is pseudo-coloured in blue, the transport vesicles in green and the Golgi apparatus in red.

fusion - did not display the same phenotype. Furthermore, blocking anterograde transport did not have the same effect. This indicates that the back-fusion phenotype is specific for tip20-8 mutants.

To provide more direct evidence for back-fusion of COPII vesicles with the endoplasmic reticulum, Kamena and Spang used a biochemical assay; in a large-scale budding experiment back-fusion occurred specifically in tip20-8 mutants. Furthermore, examination of the endoplasmic reticulum by immunofluorescence microscopy showed that back-fusion was not the result of a fragmented endoplasmic reticulum.

These findings demonstrate that an active mechanism discriminates between anterograde and retrograde vesicles in the secretory pathway. But how does Tip20 prevent back-fusion? It seems likely that Tip20 directly recognizes coat proteins, as it displays genetic interactions with multiple coatomer subunits; although, how it promotes COPI vesicle fusion while preventing COPII vesicle fusion is less clear.

JON REYNOLDS 